\title{
Editorial Highlights for Journal Rare Disorders: Diagnosis \& Therapy
}

\section{Sophia Roberts}

Managing Editor, Journal of Rare Disorders: Diagnosis \& Therapy, E-mail: raredisord@imedpubjournal.org

\section{Editorial}

I am pleased to introduce Journal Rare Disorders: Diagnosis \& Therapy (IPRDDT) is an open access Scientific Journal which mainly focuses on articles of rare and chronically debilitating diseases. I am pleased to announce that, all issues of volume 6 were published online well within the time and the print issues were also brought out and dispatched within 30 days of publishing the issue online during the year of 2020.

The aim of this journal is to produce high quality articles related to Clinical genetics and genomics, Orphan Drugs, Rare bone diseases and skeletal dysplasias, Rare cancers, Rare endocrinological diseases, Rare gastrointestinal diseases, Rare immune deficiencies, Rare systemic diseases, Haematology and Rare kidney diseases.

All published articles of this journal are included in the indexing and abstracting coverage of Index Copernicus, China National Knowledge Infrastructure (CNKI), Directory of Research Journal Indexing (DRJI), Publons and Secret Search Engine Labs.

During the calendar year of 2020 , IPRDDT received a total of 24 manuscripts, out of which 1 articles (10\%) were rejected in the preliminary screening due to plagiarism or being out of the format and peer review process. During 2019 around 17 articles were subjected for publication after they are accepted in the peer review process. In the 6 issues of volume 5 published during the year 2020, a total of 17 articles were published. Articles were published from authors all around the world. Globally articles have been accessed by the research scientists and also cited.

During the calendar year 2020, a total of three Editors, ten reviewers joined the board of IPRDDT and contributed their valuable services towards contribution as well as publication of articles, and their valuable reviewer comments will beneficial to publish quality of article in the journal.
I take this opportunity to acknowledge the contribution of (Dr. Shriram H. Bairagi) during the final editing of articles published and the support rendered by the editorial assistant, in bringing out issues of IPRDDT in time. I would also like to express my gratitude to all the authors, reviewers, the publisher, the advisory and the editorial board of IPRDDT, the office bearers for their support in bringing out yet another volume of IPRDDT and look forward to their unrelenting support to bring out the Volume 7 of IPRDDT in scheduled time. 